\title{
What Users Do: SA\&D with the ATSA Method
}

\author{
Robert B. K. Brown \\ University of Wollongong \\ bobbrown@uow.edu.au
}

\author{
Ian C. Piper \\ University of Wollongong \\ ian@uow.edu.au
}

\begin{abstract}
Incomplete or inefficient elicitation, comprehension and transmission of client requirements are all sources of Information System (IS) failure rates. Requirements may be missed, misunderstood or miscommunicated for the lack of a single, consistent, informing theory. Structured requirements elicitations techniques impose time delays. Cursory techniques can fail to reach any mutual understanding with the stakeholder. Formal methods can fail to cope with non-functional requirements and coder-oriented methods can put the cart-before-the-horse, delivering something other than required. Agile methods can deliver hasty product, cobbled to meet first-cut requirements, perhaps justified by a notion that users cannot reach stable conclusions.

This paper introduces the Activity Theoretic Software Architecture (ATSA) method, which deploys Activity Theory (AT) as it's informing theoretical framework in order to address complex human 'doings' in a non-burdensome way, to meaningfully capture requirements and generate feasible specifications with some confidence as to fidelity and consensual agreement.
\end{abstract}

\section{Keywords}

Systems Analysis and Design, SA\&D, Activity Theory, innovative method, requirements failure rates, ATSA

\section{INTRODUCTION}

Incomplete or inefficient elicitation, comprehension and transmission of client requirements are all sources of Information System (IS) failure rates. Requirements may be missed, misunderstood or miscommunicated for the lack of a single, consistent, informing theory. Decades of consistently poor results often attributed to requirements or communications issues; strongly indicates high level misunderstanding and/or misrepresentation of user requirements. It seems developed products are, too often, not what stakeholders and users actually want or need.

The 'elephant in the systems development room' is broadly acknowledged, but politely obscured, unacceptably high system failure rate. The CHAOS report (Standish, 1995) suggests that over 30\% of projects are not completed at all, that over $50 \%$ of projects run into significant cost overruns and that as few as $17 \%$ of projects are completed both on time and budget. CHAOS'95 reported that poor requirements and a lack of user involvement were the highest perceived factors. The OASIG survey (1995) produced the infamous ' $70 \%$ failure' result. Over the years many studies have reported comparable failure rates across the information systems domain.

Such infamous statistics (particularly from the 1990's) have been marginalized as outdated, however, to date no statistics have been published suggesting any significant improvement. Crear (2009), CIO of the Standish Group, reports that the failure rates in 2009 "are low point in the last five study periods. [2009's] results represent the highest failure rate in over a decade."

\section{Towards an improvement}

Approaches sensitive to the user viewpoint are valid as stakeholders generate their own notations and terminologies, complicating elicitation (Sommerville et al., 1998). Significant risk of failure exists in marginalizing stakeholder's softer objectives, despite their inherent informality. If poor requirements are at least partially attributable to poor communications between phases, analysts, designers and users; then a case exists for a lightweight, readily learnable, methodologically flexible end-to-end approach, under a single theoretical framework (addressing user activity) that concentrates on the identification of requirements. Observing the shift of focus from technology to people under user-centric design, Constantine and Lockwood (1999) said "It is not users who must be understood, but usage." Räsänen and Nyce (2006) argued for anthropological analysis to avoid skewing the focus of analysis to individual users over their larger sociostructural processes. A roughly taxonomy of evolving centrisms might be given as: Product centric, Process centric, Goal centric, then User centrism, which itself may be conceived as containing; Dependency centrism and Motive or Activity centrism. 
Perhaps the most widely studied and respected motive-activity centric approaches to the issue of requirement elicitation were based upon the psycho-social principles of Activity Theory (AT). It is well beyond the scope of this paper to properly introduce AT, so the interested reader is directed to earlier publications (AUTHOR, 2006).

AT identifies an activity as the smallest meaningful task carried out by a human subject. Vygotsky (1978) states that all human Activity is carried out by a Subject, using physical or psychological tools to achieve some object which may result in a physical outcome. Engström (1987) described the structure of an activity as a seven node matrix (Figure 1). Traditionally, AT is concerned with the cognitive ramifications of the differences between intended object and resultant outcome.

A number of Activities may reside near one another and interact, forming a network that describes a larger process (Kuutti, 1991). The outcome of one activity may constitute (among other things) a tool in another (Vrazalic, 2004). We are specifically interested in outcome-tool transactions. It is necessary to shift focus away from the psychosocial and cognitive aspects to the investigation of the facilitating tool(s) of an activity, as a subset of these could specify some new system.

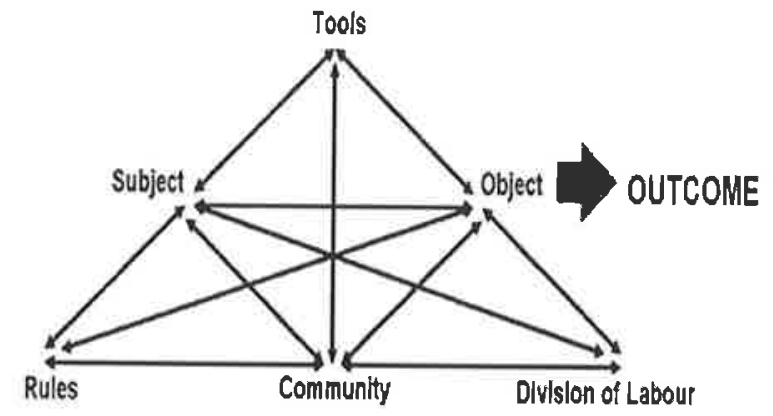

Figure 1: Engström's seven-node Activity Matrix (Engström 1987)

Ultimately, the system may be specified by describing those outcome-tool transactions and transformations which may between activities. The designer must identify and describe them. These descriptions specify the requirements for functions to facilitate these transactions and transformations. We hope to isolate those which could usefully pass through some facilitating computer system, and we will use the term 'Instrument' to refer to data-artefacts that are passed in such transactions.

Leont'ev (1978) proposed a three layer hierarchic structure: activity, action and operation to represent different levels of intellectual 'engagement' of the subject, with an activity requiring deep engagement while an operation is virtually autonomic. Kuutti (1991) introduced a topmost abstraction: the activity network.

AT was not readily convertible into a workable SA\&D method however. Martins and Daltrini (1999) unhappily reported that AT had not yet delivered any prescriptive methods. Otwell (2005) bemoaned a lack of prescriptive method for applying AT; saying "Activity Theory seems to almost defy practical application". Otwell specifically cited Collins' Activity Centered Design (ACD) work of 2002 as offering no concrete example of AT in use for design. Author (2010) surveyed thirteen attempts to apply AT to some or all of the SA\&D process and found that most were abandoned, converted into niche tools or, at best, delivered little more than a set of issues for systems designers to consider.

\section{Communication Issues}

It must be acknowledged that although a system may not be what a client wants, it could still be what they need. Such tensions could result in the 'failure' of an otherwise technically excellent product. There are grounds to suspect that SA\&D methods to date have not bridged clients' inadequate grasp of IS and the analyst/designers' inadequate grasp of their client.

Taking the broadest brush abstraction of the SDLC, we consider briefly the Analysis of what will be needed, the Design of a System to achieve that, and its Implementation. During any Analysis of 'what to make', there will be issues of translation between the analyst and the customers' paradigms. These continue as requirements are passed between the disciplines of Analysis and Design. Assuming that a viable Design can be arrived at, there will be issues of transcription between the Design and Implementation phases conveying the instructions 'make it like this'.

We propose that a common framework, with a single set of coherent concepts and a consistent terminology, would offer significant benefits.

\section{A SINGLE FRAMEWORK}

We now describe the Activity Theoretic Software Architecture (ATSA) method. ATSA commences with early phase requirements elicitation, generates an activity-based Business Process Model (BPM), then facilitates the specification of a System (including the structure of its interfaces), which would facilitate the clients' 'doings' in a feasible manner. 
The word 'architecture' was chosen to reflect the level of abstraction the method operates at. Drawing inspiration from HCI literature, the designed system 'product' was envisaged as existing in, and being complimentary to, some information/activity space. An ATSA design considers how the users interact with each other in such a space, somewhat analogous to classical architectural considerations of structural built environments.

\section{A Stepwise Description of ATSA}

The three phases of the ATSA method, with their component steps are as follows:

\section{Elicitation Phase}

\section{Identify Positions}

The analyst obtains a listing of positions in the stakeholder group to identify all possible users of the system to be designed.

Businesses may be expected to have some organization chart, and positions may be read off from there. Stakeholders must be consulted to ensure accuracy and currency. If no chart exists, the analyst may facilitate one. Positions must closely correlate to group members as they appear in their hierarchic organizational arrangement.

If there are multiple instances of a position, then the analyst may consider one exemplar of each type. Careful note must be made of all instances, as deeper analysis may reveal differences. Even under genuine duplication, multiple instances must be recorded as the system may need to handle multiple simultaneous stations, logons and possibly segregated data storage. The final system may require some form of overseeing function, assigned to a more senior user, and this might not have been indicated in the original organization chart.

\section{Identify Roles}

The analyst identifies a listing of roles. Business positions usually comprise several separately identifiable duty-based roles; though not all businesses identify them formally. Roles may be suggested by position descriptions, as retained for personnel management purposes. Role variations may be strong indicators that otherwise seemingly identical positions are different. Variations, from role information, require reclassification of positions, adopting some conveniently traceable nomenclature to differentiate them.

Roles should correlate closely to the subjects (protagonists) of activitics. Unfortunately, a group's role classifications are considerably more likely to be somewhat arbitrary duty-based classifiers, more indicative of responsibilities than of actual doings. Whilst it is very useful to gather this data, the Analyst must keep in mind that these roles may, ultimately, become less applicable or meaningful as the group's agenda and process becomes recast into AT terms. It is well worth understanding to whom each position reports or answers, but to design a feasible system it will be far more important to know what resources they require to perform their work (from whom they obtain them, and under what conditions) and what resources become available for others, as a result of their work.

The analyst may need to identify roles from scratch through interview and observation. Rather than a setback, this situation should be viewed as an excellent opportunity to elicit doing-based information rather than refine or correct somewhat arbitrary duty statements. Roles identified by the analyst are more likely to remain current through the ATSA method, and may even survive with close correlation to the final work stations of the system.

\section{Identify Candidate Instruments}

The analyst identifies 'Candidate Instruments' (CIs) from existing files, folders, forms, records, registers, lists, databases. These are data-like things (tangible or not) which roles require to perform their work, or which are made available for other roles as a result of their work. CIs may exist as formalized document types within the group.

Corporate templates or pro-forma items should constitute separate (though related) CIs to each instance or record that conforms to such a template. To do the work of creating a record, for example, would require reference to the template as well as the information to be recorded. A 'copy' of the template, in this case, enters a 'doing' as a resource and may be 'consumed' by it (being transformed into a record by some 'work'). The original template will not have been destroyed. The updating or editing of the template itself is an entirely separate administrative doing, which consume it and produce a new template, subsequently available for consumption by other roles.

The analyst should strive to avoid the temptation to define parent-child hierarchies between candidate instruments. There should only be resources and products. Container-like CIs, such as folders or files Instruments can be subsumed into 
some constraint statement, imposing (for example) an obligation or procedure which labels, stores or restricts access. Other complex CIs may be re-expressible as doings.

The analyst may identify any number of intangible CIs such as requests, permissions, state flags or conditions. Others may exist in the tacit knowledge of skilled operators. The broad elicitation questions "what do you need to perform your task" and "what do you produce or provide to others" must be pursued thoroughly to elicit as many conditions, sequences, implicit or tacit data as possible. Even a verbal call between workers may prove to be a crucial timing flag. All such intangible resources (and products) must also be recorded as they may need to be represented in some form as data objects, doings or constraint conditions.

\section{AT Analysis Phase}

In this phase the analyst recasts details of the group's process into doing-oriented terms and assembling an activity oriented BPM.

\section{Identify Goal Driven Actions}

The analyst identifies Goal Driven Actions (GDAs), each performed by some position (acting in a role), requiring some CIs, performing some work (under conditions and constraints, and to some purpose) and then producing some CIs for other GDAs to use. As initially identified, GDAs are rather short-lived. They will shortly be split up and their elements conflated into true AT activities. GDAs are convenient loci for temporarily grouping the transactions of CIs between roles.

GDAs may have multiple CIs entering and leaving. There should be only one single protagonist (actor) which will correspond closely to a position acting in a particular role. If convenient, GDAs may be represented as nodes of a directed graph, with CI transactions as arcs. Reconfiguration and rearrangement of the GDA network iteratively, under consultation with the stakeholders, yields consensual agreement and an initial BPM result.

More important is the identification of each action's goals. Under the ATSA method, an action goal is a set of statements that detail what is done and why. They list the required components (candidate instruments incoming from other actions), the work that is done to, upon or with them, and the resulting candidate instruments. Any constraints and conditions must be faithfully recorded, especially those pertaining to the timing or sequencing of doings (temporal) or to do with obligation, permission or authorization (deontic). These goal statements must be as clear and unambiguous as possible, as they are a kind of genetic information which will carry across to more refined interpretations of the network.

\section{Decompose Actions to Single Instrument Nodes (SIN's)}

The analyst then decomposes GDAs into Single Instrument Nodes (SINs). Each SIN will have only one single Instrument attached, either entering, or leaving. Each SIN inherits information from its parent's goal to explain what the instrument is for, with whom it is transacted, and its relationship to other SINs. Temporal or deontic constraints must convey constraints pertaining between the instruments involved with the parent GDA. GDAs effectively disappear at this stage, as SINs will be recombined to form activities. To with interface components however, it is necessary record each SINs' $l i$ neage.

As apparent inconsistencies and gaps arise, as is likely when a system is either undocumented or is being reinterpreted into a new conceptual framework, there may be a powerful temptation for the Analyst to indulge in re-design. Detailed piecewise deconstruction of Goal Directed Actions (GDAs) into SINs should go some way toward revealing duplications, inefficiencies and ambiguities; awareness of which can strongly provokes consideration of possible changes. Such apparent opportunities are more likely to be (or should be treated as) indicators of the need for further iterative consultation, elicitation and clarification. After all, it must be remembered that the extant group process does work in its current form to some degree, though of course there is always scope for improvement and the group itself will have recognized that or else they would not have engaged an analyst to design a computerized facilitation system.

It is necessary to strive for a complete picture of the extant group process. The analyst should therefore, strive to avoid making design changes at this time, but should feel free to note any and all issues and record any ideas towards the upcoming redesign phase.

\section{Conflate Activities}

The analyst identifies activities by conflating of SINs according firstly to the commonality of their subject (protagonist) and then the logical consistency of goals under a single motive (object).

The Analyst must be prepared to redefine roles as necessary, so long as it is the same individual user (position) performing as subject of the conflated activity.

The Analyst must consider the goal statements of the SINs and sort for those which could be logically collected under a consistent motive statement. Until some future refinement of the ATSA method incorporates a rigorously formal multimodal logical consistency checking technique, this will require some careful consideration on the part of the analyst, who would most probably benefit from consultation with the stakeholder. 
Conflation by goal consistency may give rise to further redefinition or clarification of roles. The analyst must be prepared to conduct these two sorting processes iteratively, as required. Conflated activities may closely resemble the initially identified actions, but there is no guarantee of this. The analyst must be prepared for considerable variance from the pattern of initial actions. Should the final activity network closely resemble the initially proposed actions, then the analyst has still achieved both a confirmation and the assemblage of logical constraints which accurately model the instrument transactions of the process.

It is vital that the conflated motive for each identified activity inherits all the constraints and conditions of its component SINs in a consistent manner. Any conflated activity must contain full details of its doings, of all required Instruments and of all transformations and products.

The set of activities conflated can be illustrated as a directed graph of the activity network. Each node will now be a fully formed activity which could be described according to its Engström matrix elements. Directed arcs represent instrument transactions. The adjacency matrix of this digraph is the Combined Activity Table (CAT). As a bonus outcome, this refined activity oriented BPM is of considerable use for businesses seeking quality management accreditation.

The BPM provides a mutually comprehensible tool for the stakeholder and analyst to examine the entire group process. It facilitates arguing the case for designing a central enabling computer system. There should be something of a potential void near the centre of the network where such a System could be. Inefficiencies, duplications, multiple handlings and redundancies in the current process should be highlighted and possible solutions discussed.

\section{Re-Design Phase}

Here the analyst now takes the part of a designer. Although traditionally the project might be handed off to a designer at this stage, ATSA allows for the entire SA\&D to occur under one consistent paradigm, and strongly advocates unifying analysis with design. Under consultation with the stakeholder, the designer seeks to reconfigure and rearrange the group's process to enhance efficiency through the agency of a central facilitating computer system. Once the stakeholders are accepting of a design, the method will describe it in sufficient detail to specify its requirements for detailed software-code and interface construction and implementation.

The result of this third phase is a redesigned BPM, now featuring a specification for a central facilitating computer system. The system design will be well suited to the users' doings which should positively effect usability, user acceptance and thus success. With consultation between the analyst-designer and stakeholders, the arrangements of Individuals in positions and roles may have changed, but this should only result in a closer match of the system to the group's agenda.

The system will be specified in terms of its instruments and their transactions (analogous to data in, data out and transforms) and the clustering of its interfaces according to users' doings.

\section{Run Node-Reduction Heuristics}

If the stakeholder agrees that any inefficiencies, duplications, multiple handlings and redundancies exist in the current process then a redesign of the process is indicated. This may involve redefining numerous roles and reassigning tasks (Instrument transaction flows). It is strongly recommended that the designer retain the structure of positions (people) unless the stakeholder specifically requires changes at that level.

Irrespective of changes to which doings are performed by whom, the BPM should be rationalized and modified to represent the nature and behavior of the System.

The designer should identify pipes, which are those arcs (instrument transactions) between activities which have a common subject (owner) whereby the subject sends something to themselves; and then joints which are nodes (activities) through which an instrument passes unchanged.

Locations on the activity network (BPM) where pipes and joints coincide are strong candidates for collapse and or redesign. The designer must pay particular attention to the elicited constraints (purpose, sequence, obligation etc) to ensure any modification is not in breach of the agenda.

Where necessary, activities may be split, joined or created. Whenever this occurs, their component SINs must be considered. As in previous stages, though SINs may be moved, duplicated or deleted as required, their genetic identities must be retained.

A space between the activities where some facilitating computer system could be should become clear. Many (hopefully, most) activities could be seen to be tangential to this system, able to exchange their candidate instruments through or with it. 


\section{Bend Instrument Paths to System}

As boundaries of the system become apparent, the designer reroutes instrument transactions through it. Consider that true instruments reside inside the System's Data Repository (SDR). Activities around the system boundary exchange data with the System rather than directly with each other. Following this, some activities may not to conduct any transactions across the system boundary. These peripheral activities cease to be part of the system. Details of peripheral activities should be retained however, to provide useful context and to facilitate any future revision or redesign.

Objects which are stored in the SDR are deemed to be true instruments which closely correspond to data objects and variables, but ATSA deliberately avoids such labels retain paradigmatic neutrality. ATSA outputs feasible, useful and acceptable high level specifications of the system and its interface, such that the stakeholders comprehend the product, users comprehend their tasks and builders may apply whichever techniques and languages they deem best.

\section{Convert Temporal Rules into Deontic DivLAB's}

Activities should retain constraints inherited from their component SINs. SINs rules consist largely of temporal constraints such as 'must do during state S'. Rewrite as many rules as possible into deontic DivLAB statements such as 'run iff Instrument 'state S' = TRUE'. DivLAB statements list of prerequisite instruments and their required states or values.

DivLAB constraints oblige the system to maintain and monitor instrument states, whilst rules oblige instrument consumers to ensure the correct state. The degree to which rules are converted into DivLAB's is therefore an indication of system reliability DivLABs may be coded directly. Many of SINs will become receivers. New SINs may be created, and others rewritten.

\section{Convert Goal Statements to Pseudocode}

Goal statements within each activity must be expressed as succinctly and accurately as possible. A reduced vocabulary form of structured language be employed, perhaps a form of generic pseudocode. The object (motive) of each activity will then be a logically consistent, highly structured, set of goal statements in functional requirements form.

\section{Instrument List}

There is value in sorting the specification by its instrument rather than by its activities. The coder, especially one who follows an object-oriented paradigm, is more likely to grasp the design on a per-data-object (variable) basis.

A database of some kind could prove a useful way to present this information, as it would allow the design to be sorted in various ways, and would retain activity information with each instrument. Each instrument record would offer a meaningful name and some classification of its functionality defined by the transforms it must undergo and the uses to which it is put as a resource.

It is crucial that each instrument's transactions are easily traceable through the activity network. It is important to wire up the transactions correctly, clustering Instrument handling interface widgets according to station.

\section{Identify Switches and Combine them into Screens etc}

Whilst the functions of the system are described through the instrument list and goals statements (indicating what data objects must move in and out of the system, under what transforms and constraints), the specified nature of the interface must also be generated.

Each SIN will handle a single terminus of an instrument transaction. These will cross the system boundary at the interface. The ATSA method should provide an interface designer with a sufficiently detailed description of who is handling what, and to what end, that (according to the builder's adopted paradigm) a selection of screen widgets (switches) becomes apparent. Once again, the ATSA method seeks to supply high-level, paradigm neutral specification to support a wide range of options for the builder.

Whilst much has been written, under various paradigms, about the selection of widgets and color schemes etc, an important element of interface construction that is not well understood in the literature [Dix et al. 2004] is the clustering of switches for best use. It is recognized that the assemblage of widgets can greatly impact usability, acceptance and thus success of a system.

Under the ATSA method, interface stations are assembled according to the coincidence of activity subjects (protagonists) in individual users (positions). Each station constitutes one or more screen sets, one per activity. That is to say, the interfaces are marshalled on a per activity basis. There should be no need for the user to be clumsily swapping between modes or usage instances if the interfaces have been clustered according to what they need to do.

Each activity's screen-set may contain a number of screens (windows, instances, tabs etc). Each of these should correlate to an action. Actions are no longer clearly defined at this stage however, but may be understood to be sets of SINs (sub

I This is the Activity Theoretic division of labor construct. 
sets of activity) clustered according to a common goal. Some indicator of the likely location of actions may be obtained from the genetic lineage of each SIN. It must be noted that action clustering may have changed as the BPM was redesigned to accommodate the facilitating system. It is at the level of screen clustering that ATSA still retains the greatest flexible capacity for the builder's personal intuition. More rigorous techniques for determining action (screens) from the specification may emerge under future research, perhaps drawing upon logical analysis of deontic and temporal constraints.

\section{Conclusion}

Perhaps not unexpectedly, the construction of an innovative AT based method during this research proved difficult in the extreme as the precepts of AT are famously difficult to tie down and deploy prescriptively. After numerous false starts, a set of techniques were arrived at which unexpectedly showed promise of applicability beyond HCI. Indications were that this innovative Activity Theoretic Systems Architecture (ATSA) method might serve for the analysis and design of information systems in general, inclusive of basic requirements for their user interfaces. Thus, having come full circle, AT showed indeed promise as an informing coherent basis across which systems analysis and design might be conducted.

The ATSA method was designed in strict adherence to the precepts of AT and with a view to addressing issues of coherence, communication and ease of use by the inexperienced. To ease acceptance and usefulness ATSA was intended to be agnostic of pre-existing methods and not unfriendly towards as many implementation technologies as possible. In ongoing research ATSA shows considerable promise for producing UML-style specifications deployable under object orientation, and may yet offer hope for reconciliation between 'procedural' and 'data-centric' methods.

As tested thus far in case studies and undergraduate classes, (paper in preparation) ATSA allows analysis of users' requirements and procedural specification, in some detail, in terms which a coder might readily instantiate. AT can serve as a coherent theoretical basis for systems analysis and design, inclusive of its interface requirements. Hopefully, some positive steps towards redressing some challenges which continue to face computer systems analysis and design, and that of their user interfaces, will result. The ATSA method is herewith presented for consideration, testing, development, teaching and, hopefully, application. 


\section{REFERENCES}

1. Standish Group, The (1995), The CHAOS Report (1994), [online] available from http://www.standishgroup.com/sample_research/chaos_1994_1.php [accessed 24 March 2010]

2. OASIG (1995) [cited online] in http://www.itcortex.com/Stat_Failure_Rate.htm\#The\%200ASIG\%20Study\%20(1995) Rudin, W. 91976) [accessed $15^{\text {th }}$ March 2007]

3. Crear, J. (2009), CIO Standish Group. [online] Available from http://www1.standishgroup.com/newsroom/chaos_2009.php [accessed 24th March 2010]

4. Sommerville. I., P. Sawyer, and S. Viller, (1998), Viewpoints for requirements elicitation: a practical approach, Proceedings of the 3rd IEEE international conference on requirements engineering (ICRE'98), Colorado Springs, USA, pp.74-81.

5. Constantine L. L. and Lockwood, L. A. D. (1999), Software for Use a Practical Guide to the Models and Methods of Usage-Centred Design, AddisonWesley.

6. Räsänen, M. and Nyce, J. M. (2006), A New Role for Anthropology? - rewriting 'Context' and 'Analysis' in HCI, in Nordic Conference on HumanComputer Interaction: Changing Roles (NORDICHI'06), Oslo, Norway, Proceedings New York, NY, USA. ACM Press pp. 175-184.

7. Author 2006 [redacted for review]

8. Vygotsky, L.S (1978), Mind in Society Harvard University, Press, Cambridge, MA.

9. Engström, Y.(1987), Learning by Expanding: an activity-theoretical approach to developmental research, Orienta-Konsultit Oy, Helsinki, Finland.

10. Kuutti,K.(1991) Activity Theory and its applications to information systems research and development, in Nissen, H.E., Klein, H.K., \& Hirsheims, R. (Eds) Information Systems Research: Contemporary Approaches and Emergent Traditions, Elsevier Science, Amsterdam, pp.529-549.

11. Vrazalic, L. (2004) Towards Holistic Human-Computer Interaction Evaluation Research and Practice: Development and Validation of the Distributed Usability Evaluation Method. PhD Thesis, University of Wollongong, Australia.

12. Martins, L. E. G. and Daltrini, B. M. (1999) An Approach to Software Requirements Elicitation Using Precepts from Activity Theory. in 14th IEEE International Conference on Automated Software Engineering, pp.15-23.

13. Otwell, A. (2005), Activity Theory and User-centered Design. Blog: heyblog: A Space for half-formed Thoughts. (July 29, 2005). [online] 
http:/www.heyotwell.com/heyblog/archives/2005/07/activity_theory.html [accessed 20 ${ }^{\text {th }}$ August 2008]

14. Brown R.B.K. (2010), the ATSA Method for Specifying both System and User Interface Requirements: An Application of Activity Theory, $\mathrm{PhD}$ Thesis, University of Wollongong, Australia. 